\title{
Treatment of aneurysms of the extracranial carotid artery: current evidence and future perspectives
}

\author{
GJ de Borst*, V.E.C. Pourier \\ Department of Vascular Surgery, University Medical Center Utrecht, Utrecht, The Netherlands
}

Article Info

\section{Article Notes}

Received: August 04, 2016

Accepted: September 05, 2016

\section{${ }^{*}$ Correspondence:}

Prof. Dr. Gert Jan de Borst MD PhD, University Medical Center Utrecht

Department of Vascular Surgery G04.129

PO Box 85500, 3508 GA Utrecht

The Netherlands,

Telephone: +31-88-7556965;

Email: G.J.deBorst-2@umcutrecht.nl

(C) 2016 de Borst GJ. This article is distributed under the terms of the Creative Commons

Attribution 4.0 International License

\section{Keywords:}

carotid artery

aneurysm

surgical resection

endovascular repair

\section{Introduction}

Although very rare, aneurysms of the extracranial carotid artery (ECAA) are important to identify and treat. Due to limitations in the literature consisting mostly of case reports and small case series, data on the incidence is lacking ${ }^{1,2}$. The most common used definition is a dilation of the carotid artery greater than $150 \%$ of the diameter of the normal (uninvolved) internal carotid artery (ICA) or common carotid artery (CCA). ECAAs are most frequently located in the ICA and the dilation may be focal and saccular or fusiform. The etiology is diverse and ranges from atherosclerosis, infection, fibromuscular dysplasia, and connective tissue disease, to traumatic or 'spontaneous' dissection ${ }^{3}$.

Most ECAA are asymptomatic and found by coincidence. The natural history of ECAA remains unclear (largely because of their rarity and because most reported ECAA are probably treated) ${ }^{1}$. Untreated, ECAA can cause compression in the cervical region causing swelling, pain or cranial nerve palsy. Embolization of thrombus from within the aneurysm can cause cerebral infarction, while the most feared risk of aneurysm rupture is probably very low ${ }^{2}$.

Some authors have suggested a poor natural course, with a reported stroke rate of up to $50 \%{ }^{4,5}$. Others believe that asymptomatic ECAA could be treated with a wait-andsee policy or best medical treatment ${ }^{2,4}$. These suggestions are mainly expert opinions based on clinical experience while sound scientific data is lacking. Surgical resection of the aneurysm, with arterial reconstruction, is considered by many to be the gold standard for symptomatic ECAA ${ }^{2,6}$. For ECAA due to a traumatic etiology, endovascular stenting has been advocated in the past decade. Although success rates of endovascular therapy are high, no long-term follow-up is yet available ${ }^{2,6}$. Aneurysms complicating dissection are pseudoaneurysms and the far majority of these do not increase in size and are not associated with any cerebrovascular symptoms and can therefore be managed conservatively?

For proper assessment of what treatment should be preferred, a better insight into the natural history and risk of complications of the different treatments is needed. To collect data on ECAA we designed the Carotid Aneurysm Registry (CAR), a prospective web-based international registry (www.carotidaneurysmregistry.com) assessing follow-up in patients with an ECAA ${ }^{8,9}$. The primary aim of this registry is to collect data on ECAA to assess the natural history of ECAA and the safety and durability of different treatment strategies. The present paper offers an overview of the recent work that was performed in our institution in the recent two years, and is a summary of the recent papers that were published on management of extracranial carotid artery aneurysms.

\section{Epidemiology}

Aneurysmal dilatation of the extracranial carotid artery was first reported in the late $17^{\text {th }}$ century. A recent meta-analysis has identified 255 published studies describing the management of 1279 patients; ECAAs comprise $0.6-3 \%$ of all reported carotid procedures ${ }^{2}$, but they account for only $0.4 \%$ of all peripheral aneurysms. 
However, it is likely that more (asymptomatic) ECAAs are now being identified (as opposed to being left undiagnosed) because of the increasing use of Computed Tomographic Angiography (CTA) and Magnetic Resonance Angiography in the investigation of patients presenting with a variety of head and neck pathologies or in the setting of screening for cerebral ischemia within Stroke centers.

\section{Etiology and pathogenesis}

In line with aneurysms elsewhere in the body, ECAAs can be true or false. The principle etiological causes of true ECAA include; 'atherosclerosis' , infection (i.e. tuberculosis, HIV), arteritis, fibromuscular dysplasia, Marfan's syndrome and medial degeneration. The commonest causes of false ECAAs include; iatrogenic , post carotid endarterectomy, post-trauma and post-dissection .

Bilateral ECAAs have been noted in up to $13 \%$ of patients ${ }^{3}$, while multiple synchronous aneurysms (especially intracranial) have been reported in $15-20 \%$ of patients with ECAA. In a small case series, histological examination of ECAAs revealed two distinct categories: dissection (abrupt interruption of the media) or degeneration (general loss of elastin fibers in the media). Degenerative aneurysms showed increased inflammation within the vessel wall compared to ECAAs secondary to dissection ${ }^{3}$.

\section{Clinical Features}

ECAA are more common in men than women with a male to female ratio of $2: 1$, while the mean age of reported cases is around 50 (range 35-68 years) ${ }^{2}$.

Presentation depends on etiology, location, and size. The main presentations include; pulsatile mass, transient ischaemic attack (TIA)/stroke (due to embolism or aneurysm thrombosis), carotid bruitor thrill,cranial nerve palsy(through direct compression), stridor and voice changes (recurrent laryngeal nerve compression, direct laryngeal compression), rupture (rare), Horner's syndrome (sympathetic nervous system compression) or swallowing problems.

\section{Diagnostic evaluation}

The purpose of imaging is to; 1) confirm diagnosis; 2) classify the nature of the ECAA (true/false) and its likely etiology; and 3) to assess its extent and anatomy in order to plan management ${ }^{8}$. Most aneurysms are diagnosed using duplex ultrasound. However, corroborative CTA or MRA is essential as it can provide valuable information on extent and whether an intervention might be appropriate. Limitations of CTA include a relative lack of sensitivity for lesions involving the carotid artery at the skull base or within the contrast-filled cavernous sinuses. Especially in these cases MRA might be preferable. MRA is also under evaluation to investigate aneurysm wall characteristics and further research is awaited to learn if vessel wall imaging can help to predict growth or intraluminal thrombus formation in the future ${ }^{10}$.
Several ECAA classifications have been based on the anatomic location of the aneurysm. In Bouthillier's classification, the cervical part is referred to as $\mathrm{C} 1$, the petrous part as $\mathrm{C} 2$, and the intracranial part as $\mathrm{C} 3-\mathrm{C} 7^{11}$ The classifications by Attigah and Malikov ${ }^{12,13}$ use the line of Blaisdell, which is a 'virtual' line drawn between the mastoid process and the angle of the mandible. Above this line, the carotid is considered less accessible by a standard surgical approach, and alternative exposure techniques and/or endovascular assistance may be needed.

\section{Indications for surgery}

In asymptomatic patients with small ECAA, conservative management may have a role. As a rule, patients with a symptomatic ECAA are considered candidates for invasive treatment ${ }^{6,8}$. Treatment aims are to relieve symptoms and/ or complications such as cranial nerve palsy or pharyngeal compression. The main goal, however, in ECAA management is to prevent thrombo-embolic complications. Naturally, in the case of rupture, there is an acute indication for intervention.

\section{Management}

Contemporary management is based upon mode of presentation, surgeon/interventionist experience, aneurysm location/accessibility and etiology. Two main management strategies are currently available. The first is conservative management (antiplatelet therapy, statin therapy, antihypertensive therapy). This is appropriate in selected patients (eg small asymptomatic aneurysms, truly inoperable cases and patients with severe life-limiting comorbidities). The second is to intervene with either an open surgical approach, a purely endovascular approach, or a hybrid open/ endovascular strategy. At present, open surgical exploration with aneurysm resection with/without direct repair or an interposition graft remains the gold standard ${ }^{2,6}$ Endovascular ECAA repair is an attractive and less invasive alternative to open repair, but only small case series have been published ${ }^{2}$.

Technical aspects of surgery and endovascular interventions

Preoperative planning is essential in treating ECAA. Accessibility must be assessed based on both clinical investigation and diagnostic imaging [Figure 1].

The patient's neck should be slightly extended and externally rotated. The carotid bifurcation, jugular vein, and ICA can then be dissected with special attention to protecting and preserving the vagus and hypoglossal nerves. Proximal and distal carotid control can be obtained by vascular clamps but, in the case of distal aneurysm extension, a Fogarty catheter might also be of help. Before the artery is clamped, the patient should be systemically heparinized to prevent thrombotic occlusion.

\section{Surgical treatment}

Open surgical options include; 1) proximal and distal 


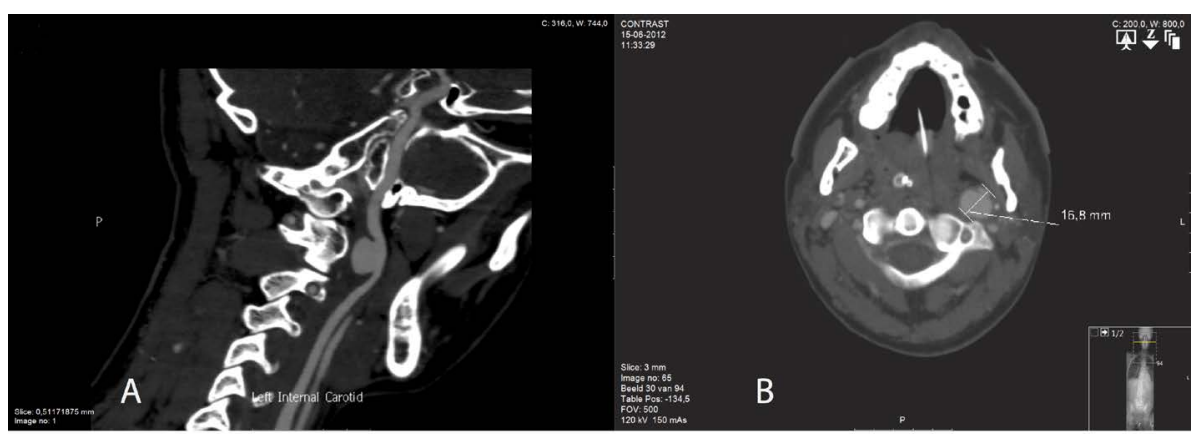

Figure 1: saccular extracranial carotid artery aneurysm, in two planes.

ligation of the ICA, 2) bypass without resection, and 3) resection of the aneurysm with direct or indirect arterial reconstruction ${ }^{6,14}$. Ligation is still used in highly selected (mostly urgent cases) such as in patients with ruptured or mycotic extracranial aneurysms. However, even if ligation may appear successful in the immediate post-operative period, cerebral infarction can still occur up to several days later.

Following sac exclusion, the ICA can be reconstructed in several ways. Where there is an elongated or redundant carotid artery adjacent to the ECAA, it is usually possible to either resect the aneurysm and perform an end-to-end anastomosis or use the proximal external carotid artery as a proximal transposition site ${ }^{6}$. Otherwise, autologous saphenous vein is the graft of choice to create an interposition bypass. Should vein be unavailable, a polytetrafluoroethylene (PTFE) or Dacron interposition graft can be used.

Sometimes the aneurysm cannot be resected or bypassed, due to its large size, extension towards the skull base or because of adherence to adjacent structures. Here it may be possible to balloon occlude the distal ICA and then perform an extra-cranial to intra-cranial bypass. Partial resection is an option in selected patients with the defect being repaired by direct closure or patch repair. This should be considered as a last resort, because it leaves diseased aneurysmal wall that will remain prone to ongoing dilatation and thrombus accumulation.

\section{Endovascular treatment}

Endovascular treatment is mostly performed with percutaneous access obtained through a common femoral artery puncture under local anesthesia. An alternate approach involves a direct puncture into the proximal common carotid artery following surgical exploration.

\section{Endovascular therapeutic options}

The stent needs to bridge healthy to healthy arterial tissue and subsequently promote thrombosis in the excluded aneurysm with continuation of blood flow to the brain. In the scarce available literature, mostly covered stents have been applied in the treatment of extracranial ICA aneurysm.
Some investigators have shown that pressure reduction and exclusion of intracranial aneurysms is feasible using bare metal stents alone or combined with coil placement. The stent must plan to cover the entire aneurysm and both landing zones must have 'disease-free' arterial walls to secure a "healthy-to-healthy" arterial bridge. Stent choice depends on arterial anatomy and aneurysmal characteristics. A wide variety of stents are available, including balloon-expandable versus self-expanding stents, bare-metal versus covered stents, and tapered versus non-tapered stents.

Bare-metal stents [BMS] are often the stent of choice for ECAA treatment. They alter the arterial inflow, which usually results in thrombosis of the aneurysm whilst preserving vessel patency. If complete aneurysm thrombosis does not occur, bare-metal stents can be combined with coil embolization. The detachable coils are inserted into the aneurysm by placing a microcatheter through the interstices of the uncovered stent. The coils alter the blood flow within the aneurysm and promote thrombus formation.

In our experience, seven consecutive symptomatic patients (4 men; mean age 52 years) with surgically inaccessible extracranial ICA aneurysms were treated with a bare stent at a single center ${ }^{15}$. All procedures were technically successful; no neurological complications occurred. After 6 months, there was complete thrombosis of the aneurysm in all except one case. In this asymptomatic patient, the residual active flow was successfully obliterated by additional coil embolization. Over a mean follow-up of $57 \pm 22$ months, all patients were alive and free of local or central neurological symptoms. All stents were patent, and thrombosis of the aneurysms was complete.

Covered stents offer an alternative management strategy in non-branching arteries and wide-necked aneurysms and in pseudoaneurysms following carotid surgery or trauma. The risk of embolisation during stent placement might be reduced through the use of a covered stent because of trapping of debris in the aneurysm that could otherwise protrude through the interstices of a bare-metal stent.

On occasions, a hybrid approach may be helpful. Here, open surgical exposure of the proximal CCA or resection of a 


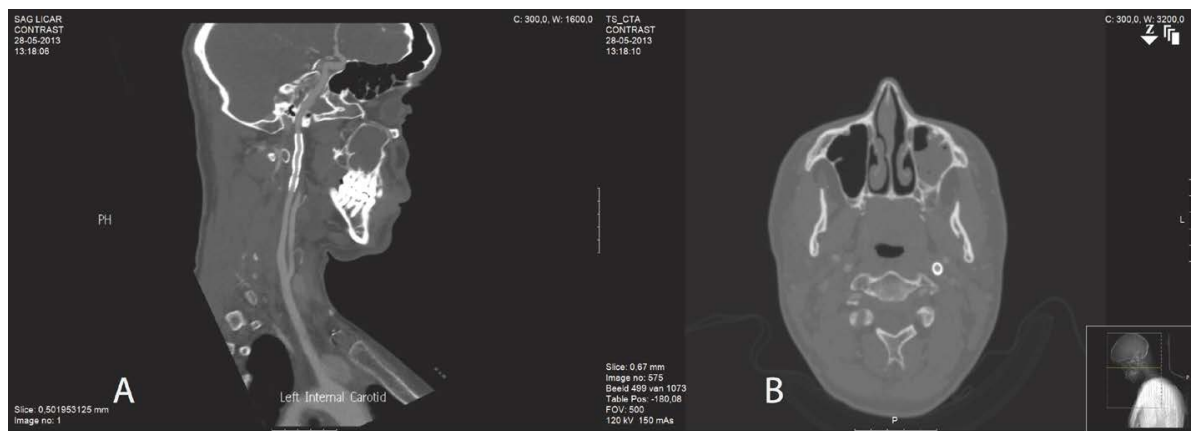

Figure 2: follow-up CT angiography following stent positiong and total exclusion of the extracranial carotid aneurysm.

loop associated with an aneurysm (followed by direct end to end arterial anastomosis), might enable the safer delivery of a covered stent into more distally located ECAAs ${ }^{2}$.

Coil embolisation is seldom employed as a first-line treatment in ECAAs because it is technically challenging, it requires the passage of a microcatheter through the neck of the aneurysm, and there is the risk of distal coil migration. Coils are therefore mainly used in combination with insertion of a bare metal stent. Embolisation of the aneurysm by percutaneous injection of thrombin is still considered too risky because of the likelihood of thrombus propagation with secondary embolisation to the brain.

One recent innovation is the flow-diverting stent, which is designed to alter blood flow in the aneurysmal vessel in favor of the longitudinal axis. In this way, it changes the inflow and outflow characteristics of blood in the aneurysm and promotes thrombosis $^{14,16}$ [Figure 2].

\section{Complications and Results}

In a recent meta-analysis, periprocedural major events occurred in $6 \%$ of patients $(2.2 \% \text { mortality, } 4.4 \% \text { stroke })^{2}$. The stroke rate was lower in endovascular treated patients (1.1\%), but 30 -day mortality was similar $(2.3 \%)$. Cranial nerve injury occurred in $0.5 \%$. Unfortunately, none of the studies have reported late outcomes.

\section{Conclusions}

The lack of an evidence-based treatment algorithm means that clinicians will have to determine individual styled management strategies for ECAA. When treatment is considered appropriate, accessibility of the ECAA is the key issue. Resection of the aneurysm with restoration of blood flow is still considered the gold standard, with acceptable procedural risks and a low risk of late stroke. Less invasive, endovascular techniques need to be further evaluated. At present, there is little or no long term follow-up data to advise on the role of these novel endovascular techniques in the management of ECAA.

\section{References}

1. McCollum CH, Wheeler WG, Noon GP, DeBakey ME. Aneurysms of the extracranial carotid artery. Twenty-one years' experience. 1979; 137, 196-200.
2. Welleweerd JC, den Ruijter HM, Kappelle LJ, Bots M, Rinkel GJ, Moll FL, et al. Management of extracranial carotid artery aneurysm. Review. EJVES. 2015; 50(2): 141-147.

3. Welleweerd JC, Nelissen BG, Koole D, de Vries JP, Moll FL, Pasterkamp G , de Borst GJ, et al. Extracranial carotid artery aneurysm can be divided in two subtypes: a histological analysis. PLoS One 2015; 10(1): e0117915.

4. Longo GM, Kibbe MR. Aneurysms of the carotid artery. Semin Vasc Surg . 2005;18:178-183.

5. Radak D, Davidovic L, Vukobratov V, Ilijevski N, Kostic D, Maksimovic Z, et al. Carotid Artery Aneurysms: Serbian Multicentric Study. Ann Vasc Surg . 2007;21:23-29.

6. Welleweerd JC, Moll FL, de Borst GJ. Technical options for the treatment of extracranial carotid aneurysms. Expert Rev Cardiovasc Ther. 2012;10(7): 925-931

7. Paraskevas KI, Batchelder AJ, Naylor AR. Fate of distal false aneurysms complicating internal carotid artery dissection: a systematic review. Eur J Vasc Endovasc Surg 2016; 52: 281-286.

8. Welleweerd JC, de Borst GJ. Extracranial carotid artery aneurysm: optimal treatment approach. EJVES 2015; 49(3): 235-236.

9. Welleweerd JC, Bots ML, Kappelle LJ, Rinkel GJ, Ruigrok YM, Baas AF, et al. Rationale and design of the extracranial carotid artery aneurysm registry. J Cardiovasc Surg (Torino) 2015.

10. Backes D, Rinkel GJ, Laban KG, Algra A, Vergouwen MD. Patient- and aneurysm specific risk factors for intracranial aneurysm growth: A systematic review and meta-analysis. Stroke 2016; 47(4): 951-957.

11. Bouthillier A, van Loveren HR, Keller JT. Segments of the internal carotid artery: a new classification. Neurosurgery. 38(3), 425-32; discussion 432-3 (1996)]

12. Attigah N, Külkens S, Zausig N, Hansmann J, Ringleb P, Hakimi M, et al. Surgical therapy of extracranial carotid artery aneurysms: long-term results over a 24-year period. Eur J Vasc Endovasc Surg 2009: 37(2), 127-133.

13. Malikov S, Thomassin JM, Magnan PE, Keshelava G, Bartoli M, Branchereau A. Open surgical reconstruction of the internal carotid artery aneurysm at the base of the skull. J Vasc Surg 2010: 51(2), 323329.

14. Pourier VE, De Borst GJ. Which carotid artery aneurysms need to be treated (and how)? J Cardiovasc Surg 2016;57(2): 152-157.

15. Welleweerd JC, de Borst GJ, de Groot G, van Herwaarden JA, Lo RT, Moll FL. Bare metal stents for treatment of extracranial internal carotid artery aneurysms: long term results. 2015; 22(1): 130-134.

16. Fischer S, Vajda Z, Aguilar Perez M, Schmid E, Hopf N, Bäzner H, et al. Pipeline embolization device (PED) for neurovascular reconstruction: initial experience in the treatment of 101 intracranial aneurysms and dissections. Neuroradiology 2011; 54(4): 369-382. 